\title{
Clinicopathological characteristics and survival outcomes in adenosquamous carcinoma of the lung: a population-based study from the SEER database
}

\author{
Jian Wang ${ }^{1, *}$, Bi Lian ${ }^{2, *}$, Ling Ye ${ }^{1, *}$, Jie Hu ${ }^{1}$ and Yuanlin Song ${ }^{1}$ \\ ${ }^{1}$ Department of Pulmonary Medicine, Zhongshan Hospital, Fudan University, Shanghai 200030, China \\ ${ }^{2}$ Department of Breast Surgery, Key Laboratory of Breast Cancer in Shanghai, Fudan University Shanghai Cancer Center, \\ Fudan University, Shanghai 200030, China \\ *These authors contributed equally to this work \\ Correspondence to: Yuanlin Song, email: song.yuanlin@zs-hospital.sh.cn \\ Jie Hu, email: hujie73@126.com \\ Keywords: adenosquamous carcinoma; adenocarcinoma; squamous cell carcinoma; prognosis \\ Received: September 07, $2017 \quad$ Accepted: October 28, $2017 \quad$ Published: December 21, 2017 \\ Copyright: Wang et al. This is an open-access article distributed under the terms of the Creative Commons Attribution License 3.0 (CC BY \\ 3.0), which permits unrestricted use, distribution, and reproduction in any medium, provided the original author and source are credited.
}

\section{ABSTRACT}

Adenosquamous carcinoma (ASC) of the lung is an unusual histology type in non-small-cell lung cancers. Due to its rarity, the clinicopathological characteristics and survival outcomes of the lung ASC are incompletely understood. We used the Surveillance, Epidemiology, and End Results (SEER) database to enroll 203,208 eligible patients, including 4,245 ASC, 124,253 adenocarcinoma (ADC) and 74,710 squamous cell carcinoma (SCC) patients. To date, this is the largest cohort in a study for ASC of the lung. With regard to age, sex, race, year of diagnosis, tumor size and SEER stage, ASC was intermediate between ADC and SCC. However, compared with ADC and SCC patients, ASC patients presented with a higher tumor grade and lower prevalence of nodal metastasis. More ASC patients underwent surgery and a lower proportion underwent radiation treatment and chemotherapy. Kaplan-Meier analysis showed that ASC patients had a better prognosis than ADC and SCC patients, but stratified analysis showed that the prognosis of ASC patients was worse than that of ADC and SCC patients in surgery and non-surgery subgroup. Multivariate analysis further confirmed that the ASC histology type was a risk factor for poor prognosis with respect to ADC and SCC. Using the propensity score matching to 1:1 match ASC with ADC or SCC, we found that ASC patients had worse survival than ADC and SCC patients. Subgroup analysis further demonstrated that ASC was a more aggressive histology type with a worse prognosis. These results provided a deep understanding of ASC, which contributed to better clinical diagnosis and treatment.

\section{INTRODUCTION}

Adenosquamous carcinoma (ASC) of the lung is defined, according to World Health Organization (WHO), as a tumor having $\geq 10 \%$ components of both adenocarcinoma (ADC) and squamous cell carcinoma (SCC) $[1,2]$. Compared with ADC or SCC, ASC is a relatively rare histology type of non-small cell lung cancers, because the prevalence of ASC has been estimated to be $0.4-4 \%$ of all lung cancers [3]. Due to its rarity, the clinicopathological characteristics and survival outcomes of ASC have not been clarified.
Differential demographic and clinical characteristics of ASC were found in different studies as for the limited case numbers. Mordant et al. [4] reported that ASC patients presented with similar features to those of ADC patients with regard to age, sex and smoking history, but had larger tumor size compared with ADC and SCC cases. However, Maeda et al. [5] showed that ASC patients were younger than ADC and SCC cases, and were intermediate between ADC and SCC patients with regard to sex. The tumor size of ASC patients was similar to that of SCC patients. Most studies have suggested that ASC patients have worse survival compared with $\mathrm{ADC}$ and $\mathrm{SCC}$ cases due to its more 
aggressive biologic behaviors, but definitive conclusions on the prognosis have not been drawn. Filosso et al. [6] reported that the 3- and 5-year survival of ASC patients after surgery were $25 \%$ and $15 \%$, respectively, and overall survival was worse than that for ADC and SCC cases. Gawrychowski et al. [7] showed that the overall survival at 5 and 10 years in ASC patients after surgery were $25.4 \%$ and $19.2 \%$, respectively, and that ASC carried a worse prognosis compared with ADC and SSC. Cooke et al. [8] identified 872 ASC patients who underwent surgery from the Surveillance, Epidemiology, and End Results (SEER) database (1998-2002), and found that ASC had a worse prognosis than ADC and SCC. However, some studies have not showed a significant difference in the prognosis between ASC and ADC or SCC [0]. In 1999, WHO reclassified histology criteria for lung ASC [1], but most cases in these studies were obtained before 1999, which resulted in confounding of the clinicopathological characteristics and survival outcomes of ASC.

So far, few scholars have attempted to identify the prognostic factors for ASC. Watanabe et al. [11] enrolled 53 ASC patients and found that tumor size $>5 \mathrm{~cm}$ and peritumoral inflammatory changes on computed tomography (CT) were independent prognostic factors for ASC. Filosso et al. [6] reviewed of $48 \mathrm{ASC}$ patients retrospectively, and identified that distant metastasis, tumor perineural invasion and tumor stage were risk factors for prognosis and that receiving adjuvant chemotherapy was a protective factor for the prognosis in ASC patients. The small number of ASC patients in these studies reduced the reliability and practical importance of their results for the clinical diagnosis and treatment of ASC. A large population-based and multi-center study to clarify the clinicopathological characteristics and survival outcomes of ASC is lacking.

In the present study, we obtained the clinicopathological and prognostic data of patients with ASC, ADC and SCC from the SEER (2000-2014), which was a large population-based database supported by the American National Cancer Institute. Differences between ASC and $\mathrm{ADC}$ or SCC were identified to make a deeper understand on ASC.

\section{MATERIALS AND METHODS}

\section{Ethics statement}

We have signed the SEER Research Data Agreement for the access to the SEER data using the reference number 11782-Nov2016. The informed consent was not required in this study because there was no personal identifying information in SEER database. This study was approved by the Ethical Committee and Institutional Review Board of Zhongshan Hospital, Fudan University.

\section{Study population}

We obtained patient data from the SEER database (Submission, November 2016). It contains research data from 18 population-based cancer registries and the Hurricane Katrina impacted Louisiana cases from 1973 to 2014, and covers approximately $27.8 \%$ of the American population. Eligible patients were selected based on the tool SEER*Stat v8.3.4 (released 23 March 2017) from the National Cancer Institute.

Patients diagnosed based on histology with lung ASC, ADC or SCC were enrolled in the present study. Inclusion criteria were shown as follows: age at the diagnosis $\geq 18$ years; site recode ICD-O-3/WHO 2008 (International Classification of Diseases for Oncology, Third Edition) was restricted to "Lung and Bronchus"; pathologically confirmed ASC (ICD-O-3 8560/3) was selected whereas the pathologic type of ADC and SCC were identified according to the criteria stated in the previous study [12]; the diagnosis was not confirmed by autopsy or death certificate; only one malignant primary tumor was diagnosed. We excluded patients who had incomplete survival data. Patients diagnosed before 2000 were also excluded because WHO updated the definition of lung ASC in 1999.

\section{Covariates}

The covariates including age, race, sex, year of diagnosis, marital status, tumor grade, tumor size, SEER stage, nodal status, surgery, radiation and chemotherapy were extracted for further analysis. We classified age into four groups: $<60,60-69,70-79$, and $\geq 80$. The year of diagnosis was classified into three groups: 2000-2004, 2005-2009, and 2010-2014. Tumor size (cm) was categorized as follows: $\leq 3,>3$ and $\leq 5,>5$ and $\leq 7$, and $>7$. As for the different criteria for American Joint Committee on Cancer (AJCC) stage in the SEER database during the study period, the SEER stage was used to describe the tumor stage. It was classified into localized, regional and distant according to the SEER program.

Cancer-specific survival (CSS) was used as the primary outcome of our study. CSS was defined as the follow-up time from the diagnosis to death due to lung cancer. The cutoff date for follow-up was 31 December 2014. Any patient who died due to other causes before this cutoff date or who was alive on the date of last contact was censored.

\section{Statistical analysis}

The demographic and clinical characteristics of ASC patients were compared with those of ADC or SCC patients using the chi-square test. The Kaplan-Meier method was used to generate survival curves. Differences between these curves were analyzed by the log-rank test. After proportional hazards assumption was checked using Schoenfeld residuals, univariate and multivariate Cox proportional models were used to identify prognostic factors and the results were shown as hazard ratios (HRs) and $95 \%$ confidence intervals $(95 \% \mathrm{CI})$. To reduce the 
effects of differences in characteristics among the three groups on CSS, a propensity score matching (PSM) method to 1:1 match ASC with ADC or SCC patients, respectively, was used. The factors for matching ASC with ADC were age, race, sex, year of diagnosis, tumor grade, tumor size, SEER stage, nodal status, surgery, radiation and chemotherapy, while the factors for matching ASC with SCC were age, race, sex, year of diagnosis, marital status, tumor grade, tumor size, SEER stage, nodal status, surgery, and radiation. PSM was undertaken using the psmatch2 module in Stata v14.0 (StataCorp, College Station, TX, USA). Subgroups analysis was done using a multivariate Cox proportional model to determine the HRs of ASC vs. ADC or ASC vs. SCC in a matched population according to covariate stratification. Grade I subgroup was excluded because the number of patients was small.

All statistical analysis were carried out using SPSS v20.0 (IBM, Armonk, NY, USA). $P<0.05$ (two-sided) was considered significant.

\section{RESULTS}

\section{Baseline characteristics of the study cohort}

The demographic and clinical characteristics of the three patient groups were shown in Table 1. A total of 203,208 eligible patients were identified: 4,245 with ASC, 124,253 with ADC and 74,710 with SCC. A significant difference between ASC and ADC cases was found with respect to age, race, sex, year of diagnosis, tumor grade, tumor size, SEER stage, nodal status, surgery and chemotherapy. Compared with ADC patients, ASC patients were older (70-79: $33.3 \%$ vs. $28.4 \%$; $\geq 80: 13.3 \%$ vs. $12.7 \% ; P<0.001)$, were predominantly male $(55.3 \%$ vs. $49.0 \% ; P<0.001)$, had more white race $(82.0 \%$ vs. $79.4 \% ; P<0.001$ ), had a higher tumor grade (III-IV: $49.0 \%$ vs. $30.9 \% ; P<0.001)$, had greater tumor size $(5$ $\mathrm{cm}<$ size $\leq 7 \mathrm{~cm}: 14.8 \%$ vs. $11.0 \%$; size $>7 \mathrm{~cm}: 8.4 \%$ vs. $7.0 \% ; P<0.001)$ and a higher proportion of ASC patients underwent surgery $(50.4 \%$ vs. $32.5 \% ; P<0.001)$. However, ASC patients had the lower prevalence of distant metastasis according to SEER stage (38.5\% vs. 53.2\%; $P<$ $0.001)$ and nodal metastasis $(50.6 \%$ vs. $51.0 \% ; P<0.001)$, and fewer ASC patients underwent chemotherapy (42.9\% vs. $47.9 \% ; P<0.001)$. There was no significant difference between ASC and ADC cases with regard to marital status and radiation treatment. When comparing ASC patients with SCC cases, a significant difference was found in age, race, sex, year of diagnosis, marital status, tumor grade, tumor size, SEER stage, nodal status, surgery, and radiation, but not for chemotherapy. Compared with SCC cases, ASC patients were younger (<60: $23.0 \%$ vs. $18.5 \%$; $P<0.001$ ), had a higher tumor grade (III-IV: $49.0 \% v s$. $38.3 \% ; P<0.001)$ and had the higher prevalence of distant metastasis according to SEER stage (38.5\% vs. $37.7 \%$; $P<0.001)$. More ASC patients were married $(54.8 \%$ vs. $51.4 \% ; P<0.001)$ and underwent surgery $(50.4 \%$ vs. 29.1\%; $P<0.001)$. However, compared with SCC cases, fewer ASC patients were male (55.3\% vs. 64.1\%; $P<0.001)$, had white race $(82.0 \%$ vs. $82.2 \% ; P<0.001)$ and underwent radiation treatment $(40.3 \%$ vs. $47.9 \%$; $P<0.001)$. They had a smaller tumor size (size $\leq 3 \mathrm{~cm}$ : $35.0 \%$ vs. $23.5 \% ; P<0.001)$ and the lower prevalence of nodal metastasis $(50.6 \%$ vs. $51.5 \% ; P<0.001)$, compared with SCC patients. In summary, compared with ADC and SCC patients, ASC patients had a higher tumor grade and lower prevalence of nodal metastasis. More ASC patients underwent surgery and fewer had radiation treatment and chemotherapy. However, ASC cases were intermediate between ADC and SCC patients with regard to age, race, sex, year of diagnosis, tumor size and SEER stage.

\section{Comparison survival among the three groups}

The Kaplan-Meier method was used to analyze CSS among these three histology types (Figure 1). Unexpectedly, ASC patients carried a better prognosis than ADC and SCC patients, respectively (ASC vs. ADC, $P<0.001$; ASC vs. SCC, $P<0.001)$. CSS at 1 , 3 and 5 years for ASC patients were $57.3 \%, 34.6 \%$ and $28.2 \%$, respectively, and all of these CSS values were higher than those of ADC and SCC patients. The SCC patients seemed to have the worst survival. Univariate and multivariate Cox proportional hazard models were used to identify the prognostic factors associated with CSS (Table 2). In the univariate analysis, all of the covariates (histology type, age, race, sex, year of diagnosis, marital status, tumor grade, tumor size, SEER stage, nodal status, surgery, radiation and chemotherapy) showed a significant association with CSS. The multivariate analysis adjusted for all of these covariates, and found that all of these covariates remained prognostic factors for CSS. Interestingly, the ASC histology type (set as the reference) was found to be a protective factor compared with ADC or SCC in the univariate analysis. However, the multivariate analysis showed that ASC patients had worse CSS than ADC or SCC patients. Similarly, chemotherapy was a risk factor for CSS in the univariate analysis but was a protective factor for CSS in the multivariate analysis. Besides, SEER stage and surgery were considered to be the most significant prognostic factors for CSS.

\section{Stratified analysis according to SEER stage and surgery}

We stratified the study population according to the SEER stage or surgery to further evaluate the difference between ASC and ADC or SCC. The Kaplan-Meier method was used to evaluate CSS based on SEER stage or surgery (Figures 2-3). In the localized or regional subgroup, ASC patients were intermediate between $\mathrm{ADC}$ and SCC cases with regard to CSS. There was no 
significant difference in survival curves between ASC and SCC patients with distant disease. However, in the surgery group or non-surgery subgroup, ASC patients had worse survival compared with ADC or SCC patients. Univariate and multivariate Cox proportional hazard models were employed to evaluate the HRs of these three histology types for CSS, and the ASC histology type was set as the reference (Table 3 ). In the univariate and multivariate analysis, the ASC histology type was confirmed to be an independent risk factor for CSS in the surgery and nonsurgery subgroup. However, different results were found in subgroups stratified according to SEER stage. In the univariate analysis, ASC patients had a worse prognosis compared with ADC patients. However, when compared with SCC patients, ASC patients had a better prognosis in terms of localized or regional disease, but there was no difference with regard to distant disease. In the multivariate analysis, ASC was an independent risk factor for CSS in the regional and distant subgroups, but no significant difference in the prognosis was found among these three histology types in the localized subgroup.

\section{Survival analysis in matched groups}

To eliminate the confounding effect from an imbalance in baseline characteristics of these three patient groups on lung cancer outcomes, we conducted 1:1 matched case-control analysis using PSM to match ASC with ADC and SCC patients, respectively. We obtained a group of 8,490 patients (4,245 ASC and 4,245 ADC patients) and a group of 7,852 patients $(3,926$ ASC patients and 3,926 SCC patients) (Table 4A-4B). No significant differences in baseline characteristics were found between
ASC and ADC or SCC patients in the matched groups. Interestingly, matched ASC patients exhibited a worse prognosis compared with matched ADC or matched SCC patients, respectively, using a Kaplan-Meier method (Figure 4). After adjustment for these covariates using multivariate analysis, ASC patients continued to have worse survival than ADC or SCC patients (ASC vs. ADC: $\mathrm{HR}=1.14,95 \%$ CI 1.08-1.20, $p<0.001$; ASC $v s$. SCC: $\mathrm{HR}=1.20,95 \%$ CI 1.13-1.27, $p<0.001$ ) (Supplementary Table 1A-1B).

\section{Subgroup analysis in matched groups}

To further elucidate the characteristics of lung ASC patients, we undertook subgroup analysis in matched groups (Figure 5). Analysis for most of subgroups showed that ASC patients had a worse prognosis than ADC or SCC patients. However, in the subgroup analysis of ASC and ADC patients, there was no significant difference for HRs for age $<60(\mathrm{HR}=1.11,95 \%$ CI 0.99-1.24, $p=0.062)$, age $\geq 80(\mathrm{HR}=1.11,95 \%$ CI $0.95-1.28, p$ $=0.188)$, black race $(\mathrm{HR}=1.04,95 \%$ CI $0.88-1.22, p$ $=0.644)$, other races $(\mathrm{HR}=1.24,95 \%$ CI $0.98-1.57$, $p=0.068$ ), year of diagnosis between 2000 and 2004 $(\mathrm{HR}=1.07,95 \%$ CI $0.97-1.17, p=0.186)$, tumor size $\leq 3 \mathrm{~cm}(\mathrm{HR}=1.06,95 \%$ CI $0.96-1.18, p=0.262)$, tumor size $>7 \mathrm{~cm}(\mathrm{HR}=1.06,95 \%$ CI $0.89-1.27, p=$ $0.500)$, and localized subgroup ( $\mathrm{HR}=1.10,95 \% \mathrm{CI}$ $0.94-1.28, p=0.220)$. In the subgroup analysis of ASC and SCC patients, no significant difference was found for HRs for age $<60(\mathrm{HR}=1.10,95 \%$ CI $0.98-1.24$, $p=0.116)$, black race $(\mathrm{HR}=1.17,95 \%$ CI $0.98-1.38$, $p=0.076)$, other races $(\mathrm{HR}=1.02,95 \%$ CI $0.78-1.33$,

\section{Cancer specific survival}

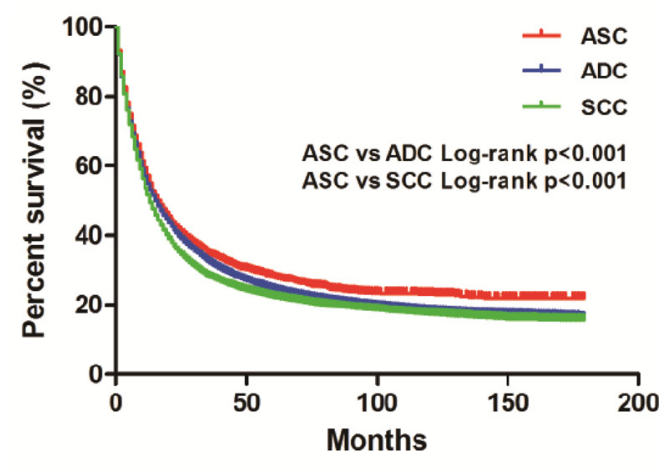

\begin{tabular}{llll}
\hline & 1-year & 3-year & 5-year \\
\hline ASC & $57.3 \%$ & $34.6 \%$ & $28.2 \%$ \\
ADC & $55.0 \%$ & $32.1 \%$ & $24.4 \%$ \\
SCC & $51.6 \%$ & $28.1 \%$ & $22.3 \%$ \\
\hline
\end{tabular}

Figure 1: Kaplan-Meier plot and log-rank test for the cancer-specific survival (CSS) among these three histological types. ASC vs. ADC, $p<0.001$; ASC vs. SCC, $p<0.001$. 
Table 1: The demographic and clinical characteristics of these three patient groups

\begin{tabular}{|c|c|c|c|c|c|}
\hline Characteristics & $\begin{array}{c}\text { ASC } \\
N=4245(\%)\end{array}$ & $\begin{array}{c}\text { ADC } \\
N=124253(\%)\end{array}$ & $\begin{array}{c}\text { SCC } \\
N=74710(\%)\end{array}$ & $\begin{array}{c}p \text { value } \\
\text { ASC vs. ADC }\end{array}$ & $\begin{array}{c}p \text { value } \\
\text { ASC vs. SCC }\end{array}$ \\
\hline Age & & & & $<0.001$ & $<0.001$ \\
\hline$<60$ & $977(23.0)$ & 34609 (27.9) & $13814(18.5)$ & & \\
\hline $60-69$ & $1292(30.4)$ & $38618(31.1)$ & $23782(31.8)$ & & \\
\hline 70-79 & $1413(33.3)$ & 35259 (28.4) & $26064(34.9)$ & & \\
\hline$\geq 80$ & $563(13.3)$ & 15767 (12.7) & $11050(14.8)$ & & \\
\hline Sex & & & & $<0.001$ & $<0.001$ \\
\hline Female & $1896(44.7)$ & $63397(51.0)$ & $26802(35.9)$ & & \\
\hline Male & $2349(55.3)$ & $60856(49.0)$ & 47908 (64.1) & & \\
\hline Race & & & & $<0.001$ & $<0.001$ \\
\hline White & $3481(82.0)$ & 98621 (79.4) & $61441(82.2)$ & & \\
\hline Black & $471(11.1)$ & $14552(11.7)$ & 9390 (12.6) & & \\
\hline Others $^{b}$ & $285(6.7)$ & $10757(8.7)$ & $3732(5.0)$ & & \\
\hline Unknown & $8(0.2)$ & $323(0.3)$ & $147(0.2)$ & & \\
\hline Year of diagnosis & & & & $<0.001$ & 0.006 \\
\hline 2000-2004 & $1192(28.1)$ & $31943(25.7)$ & $22298(29.8)$ & & \\
\hline 2005-2009 & $1352(31.8)$ & 39098 (31.5) & $24202(32.4)$ & & \\
\hline 2010-2014 & $1701(40.1)$ & $53212(42.8)$ & $28210(37.8)$ & & \\
\hline Marital status & & & & 0.39 & $<0.001$ \\
\hline Married & $2327(54.8)$ & $67271(54.1)$ & 38385 (51.4) & & \\
\hline Not married ${ }^{c}$ & $1730(40.8)$ & $51803(41.7)$ & $33219(44.5)$ & & \\
\hline Unknown & $188(4.4)$ & $5179(4.2)$ & $3106(4.2)$ & & \\
\hline Grade $^{d}$ & & & & $<0.001$ & $<0.001$ \\
\hline I & $42(1.0)$ & $9567(7.7)$ & $1819(2.4)$ & & \\
\hline II & $907(21.4)$ & 30575 (24.6) & $22651(30.3)$ & & \\
\hline III-IV & $2079(49.0)$ & $38381(30.9)$ & $28601(38.3)$ & & \\
\hline Unknown & $1217(28.7)$ & $45730(36.8)$ & $21639(29.0)$ & & \\
\hline Tumor size (cm) & & & & $<0.001$ & $<0.001$ \\
\hline$\leq 3$ & $1486(35.0)$ & $46030(37.0)$ & $17593(23.5)$ & & \\
\hline $3-5$ & 1164 (27.4) & $29313(23.6)$ & $19050(25.5)$ & & \\
\hline $5-7$ & $627(14.8)$ & $13619(11.0)$ & $12877(17.2)$ & & \\
\hline$>7$ & $355(8.4)$ & $8650(7.0)$ & $9860(13.2)$ & & \\
\hline Unknown & $613(14.4)$ & 26641 (21.4) & $15330(20.5)$ & & \\
\hline SEER stage & & & & $<0.001$ & $<0.001$ \\
\hline Localized & $985(23.2)$ & 25319 (20.4) & $16713(22.4)$ & & \\
\hline Regional & $1572(37.0)$ & 30518 (24.6) & $27878(37.3)$ & & \\
\hline Distant & $1634(38.5)$ & 66050 (53.2) & $28146(37.7)$ & & \\
\hline Unknown & $54(1.3)$ & $2366(1.9)$ & $1973(2.6)$ & & \\
\hline Nodal status & & & & $<0.001$ & $<0.001$ \\
\hline No & $1861(43.8)$ & $48591(39.1)$ & $29857(40.0)$ & & \\
\hline Yes & $2146(50.6)$ & $63330(51.0)$ & $38470(51.5)$ & & \\
\hline Unknown & $238(5.6)$ & $12332(9.9)$ & $6383(8.5)$ & & \\
\hline Surgery & & & & $<0.001$ & $<0.001$ \\
\hline No & $2091(49.3)$ & $83330(67.1)$ & $52414(70.2)$ & & \\
\hline Yes & $2138(50.4)$ & 40349 (32.5) & $21766(29.1)$ & & \\
\hline Unknown & $16(0.4)$ & $574(0.5)$ & $530(0.7)$ & & \\
\hline Radiation & & & & 0.085 & $<0.001$ \\
\hline No/ Unknown & $2592(61.1)$ & 74229 (59.7) & 38891 (52.1) & & \\
\hline Yes & $1653(38.9)$ & $50024(40.3)$ & 35819 (47.9) & & \\
\hline Chemotherapy & & & & $<0.001$ & 0.26 \\
\hline No/ Unknown & $2424(57.1)$ & $64712(52.1)$ & 42003 (56.2) & & \\
\hline Yes & $1821(42.9)$ & 59541 (47.9) & 32707 (43.8) & & \\
\hline
\end{tabular}

Abbreviations: ASC, adenosquamous carcinoma; ADC, adenocarcinoma; SCC, squamous cell carcinoma; SEER, Surveillance Epidemiology and End Results database. ${ }^{a} p$ value between $\mathrm{ASC}$ and $\mathrm{ADC}$ or SCC was calculated by chi-square test, respectively.

${ }^{b}$ Others included American Indian/Alaskan native, and Asian/Pacific islander.

${ }^{\circ}$ Not married included separated, single (never married), divorced, unmarried or domestic partner and widowed.

${ }^{\mathrm{d}}$ Grade I is well-differentiated; Grade II is moderately differentiated; Grade III is poorly differentiated; Grade IV is undifferentiated. 
Table 2: Univariate and multivariate analysis of cancer-specific survival (CSS) among these three groups

\begin{tabular}{|c|c|c|c|c|}
\hline \multirow{2}{*}{ Characteristics } & \multicolumn{2}{|c|}{ Univariate analysis } & \multicolumn{2}{|c|}{ Multivariate analysis } \\
\hline & HR (95\% CI) & $p$ value & HR (95\% CI) & $p$ value \\
\hline \multicolumn{5}{|l|}{ Histological type } \\
\hline ASC & Reference & - & Reference & - \\
\hline $\mathrm{ADC}$ & $1.09(1.05-1.13)$ & $<0.001$ & $0.87(0.84-0.90)$ & $<0.001$ \\
\hline $\mathrm{SCC}$ & $1.18(1.13-1.22)$ & $<0.001$ & $0.88(0.84-0.91)$ & $<0.001$ \\
\hline \multicolumn{5}{|l|}{ Age } \\
\hline$<60$ & Reference & - & Reference & - \\
\hline $60-69$ & $0.97(0.96-0.99)$ & $<0.001$ & $1.09(1.07-1.10)$ & $<0.001$ \\
\hline $70-79$ & $1.06(1.04-1.07)$ & $<0.001$ & $1.22(1.21-1.24)$ & $<0.001$ \\
\hline$\geq 80$ & $1.27(1.25-1.30)$ & $<0.001$ & $1.32(1.30-1.35)$ & $<0.001$ \\
\hline \multicolumn{5}{|l|}{ Sex } \\
\hline Female & Reference & - & Reference & - \\
\hline Male & $1.24(1.22-1.25)$ & $<0.001$ & $1.18(1.17-1.19)$ & $<0.001$ \\
\hline \multicolumn{5}{|l|}{ Race } \\
\hline White & Reference & - & Reference & - \\
\hline Black & $1.15(1.13-1.17)$ & $<0.001$ & $0.99(0.97-1.00)$ & 0.091 \\
\hline Others $^{\mathrm{a}}$ & $0.89(0.87-0.91)$ & $<0.001$ & $0.77(0.75-0.79)$ & $<0.001$ \\
\hline Unknown & $0.62(0.54-0.72)$ & $<0.001$ & $0.54(0.47-0.62)$ & $<0.001$ \\
\hline \multicolumn{5}{|l|}{ Year of diagnosis } \\
\hline 2000-2004 & Reference & - & Reference & - \\
\hline 2005-2009 & $0.88(0.87-0.90)$ & $<0.001$ & $0.88(0.87-0.89)$ & $<0.001$ \\
\hline 2010-2014 & $0.81(0.80-0.82)$ & $<0.001$ & $0.79(0.78-0.80)$ & $<0.001$ \\
\hline \multicolumn{5}{|l|}{ Marital status } \\
\hline Married & Reference & - & Reference & - \\
\hline Not married ${ }^{b}$ & $1.12(1.11-1.14)$ & $<0.001$ & $1.09(1.08-1.11)$ & $<0.001$ \\
\hline Unknown & $1.04(1.01-1.07)$ & 0.003 & $1.01(0.98-1.04)$ & 0.638 \\
\hline \multicolumn{5}{|l|}{ Grade $^{\mathrm{e}}$} \\
\hline I & Reference & - & Reference & - \\
\hline II & $1.64(1.59-1.70)$ & $<0.001$ & $1.34(1.29-1.38)$ & $<0.001$ \\
\hline III-IV & $2.35(2.27-2.43)$ & $<0.001$ & $1.53(1.47-1.58)$ & $<0.001$ \\
\hline Unknown & $3.51(3.39-3.63)$ & $<0.001$ & $1.44(139-1.49)$ & $<0.001$ \\
\hline \multicolumn{5}{|l|}{ Tumor size (cm) } \\
\hline$\leq 3$ & Reference & - & Reference & - \\
\hline $3-5$ & $1.67(1.64-1.70)$ & $<0.001$ & $1.23(1.21-1.25)$ & $<0.001$ \\
\hline $5-7$ & $2.20(2.16-2.24)$ & $<0.001$ & $1.42(1.39-1.45)$ & $<0.001$ \\
\hline$>7$ & $2.69(2.64-2.75)$ & $<0.001$ & $1.61(1.58-1.64)$ & $<0.001$ \\
\hline Unknown & $3.13(3.08-3.18)$ & $<0.001$ & $1.46(1.43-1.48)$ & $<0.001$ \\
\hline \multicolumn{5}{|l|}{ SEER stage } \\
\hline Localized & Reference & - & Reference & - \\
\hline Regional & $2.24(2.20-2.28)$ & $<0.001$ & $1.65(1.62-1.69)$ & $<0.001$ \\
\hline Distant & $5.95(5.84-6.06)$ & $<0.001$ & $3.13(3.06-3.20)$ & $<0.001$ \\
\hline Unknown & $3.69(3.55-3.83)$ & $<0.001$ & $1.37(1.31-1.43)$ & $<0.001$ \\
\hline \multicolumn{5}{|l|}{ Nodal status } \\
\hline No & Reference & - & Reference & - \\
\hline Yes & $2.43(2.40-2.46)$ & $<0.001$ & $1.29(1.27-1.31)$ & $<0.001$ \\
\hline Unknown & $3.11(3.05-3.17)$ & $<0.001$ & $1.26(1.23-1.28)$ & $<0.001$ \\
\hline \multicolumn{5}{|l|}{ Surgery } \\
\hline No & Reference & - & Reference & - \\
\hline Yes & $0.21(0.20-0.21)$ & $<0.001$ & $0.36(0.35-0.36)$ & $<0.001$ \\
\hline Unknown & $0.88(0.82-0.94)$ & $<0.001$ & $0.89(0.83-0.95)$ & $<0.001$ \\
\hline \multicolumn{5}{|l|}{ Radiation } \\
\hline No/Unknown & Reference & - & Reference & - \\
\hline Yes & $1.60(1.58-1.62)$ & $<0.001$ & $1.01(1.00-1.02)$ & 0.034 \\
\hline \multicolumn{5}{|l|}{ Chemotherapy } \\
\hline No/ Unknown & Reference & - & Reference & - \\
\hline Yes & $1.24(1.23-1.25)$ & $<0.001$ & $0.62(0.61-0.63)$ & $<0.001$ \\
\hline
\end{tabular}

Abbreviations: ASC, adenosquamous carcinoma; ADC, adenocarcinoma; SCC, squamous cell carcinoma; SEER, Surveillance Epidemiology and End Results database; HR, hazard ratio; CI, confidence interval.

${ }^{a}$ Others included American Indian/Alaskan native, and Asian/Pacific islander.

${ }^{b}$ Not married included separated, single (never married), divorced, unmarried or domestic partner and widowed.

${ }^{\mathrm{c}}$ Grade I is well-differentiated; Grade II is moderately differentiated; Grade III is poorly differentiated; Grade IV is undifferentiated. 
Table 3: Univariate and multivariate analysis of three histological types on cancer-specific survival (CSS) based on SEER stage or surgery

\begin{tabular}{|c|c|c|c|c|}
\hline \multirow{2}{*}{ Characteristics } & \multicolumn{2}{|c|}{ Univariate analysis } & \multicolumn{2}{|c|}{ Multivariate analysis } \\
\hline & HR (95\% CI) & $p$ value & HR $(95 \%$ CI) & $p$ value \\
\hline \multicolumn{5}{|l|}{ SEER stage } \\
\hline \multicolumn{5}{|l|}{ Localized } \\
\hline ASC & Reference & - & Reference & - \\
\hline $\mathrm{ADC}$ & $0.87(0.78-0.97)$ & 0.012 & $0.90(0.81-1.00)$ & .051 \\
\hline SCC & $1.38(1.23-1.53)$ & $<0.001$ & $0.96(0.86-1.07)$ & .430 \\
\hline \multicolumn{5}{|l|}{ Regional } \\
\hline ASC & Reference & - & Reference & - \\
\hline $\mathrm{ADC}$ & $0.91(0.85-0.97)$ & 0.005 & $0.83(0.77-0.88)$ & $<0.001$ \\
\hline SCC & $1.31(1.22-1.40)$ & $<0.001$ & $0.85(0.80-0.91)$ & $<0.001$ \\
\hline \multicolumn{5}{|l|}{ Distant } \\
\hline ASC & Reference & - & Reference & - \\
\hline $\mathrm{ADC}$ & $0.89(0.84-0.94)$ & $<0.001$ & $0.89(0.85-0.94)$ & $<0.001$ \\
\hline $\mathrm{SCC}$ & $1.01(0.96-1.07)$ & 0.656 & $0.88(0.83-0.93)$ & $<0.001$ \\
\hline \multicolumn{5}{|l|}{ Surgery } \\
\hline \multicolumn{5}{|l|}{ No } \\
\hline ASC & Reference & - & Reference & - \\
\hline $\mathrm{ADC}$ & $0.87(0.83-0.91)$ & $<0.001$ & $0.87(0.83-0.91)$ & $<0.001$ \\
\hline SCC & $0.87(0.83-0.91)$ & $<0.001$ & $0.89(0.85-0.94)$ & $<0.001$ \\
\hline \multicolumn{5}{|l|}{ Yes } \\
\hline ASC & Reference & - & Reference & - \\
\hline $\mathrm{ADC}$ & $0.72(0.68-0.77)$ & $<0.001$ & $0.87(0.82-0.93)$ & $<0.001$ \\
\hline $\mathrm{SCC}$ & $0.83(0.78-0.89)$ & $<0.001$ & $0.81(0.76-0.86)$ & $<0.001$ \\
\hline
\end{tabular}

Abbreviations: ASC, adenosquamous carcinoma; ADC, adenocarcinoma; SCC, squamous cell carcinoma; SEER, Surveillance Epidemiology and End Results database; HR, hazard ratio; CI, confidence interval.

A

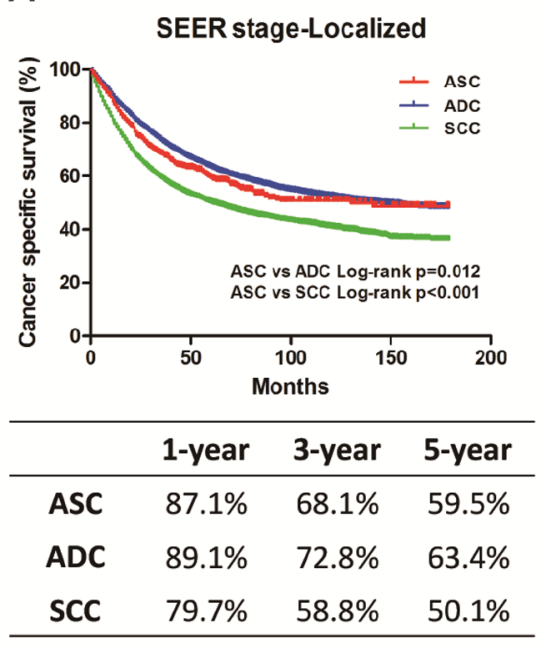

B

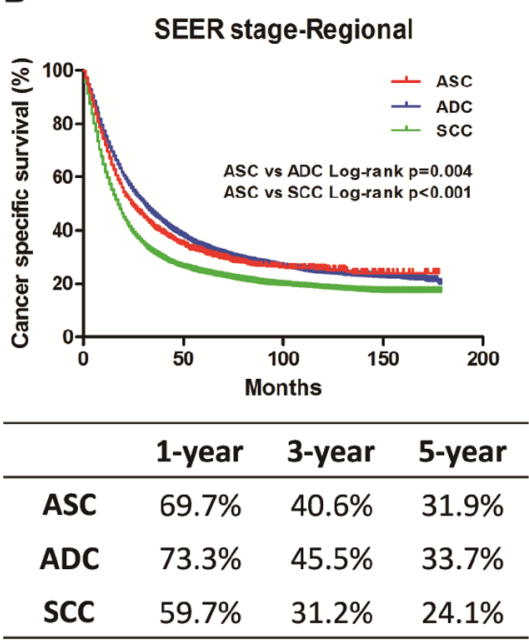

C

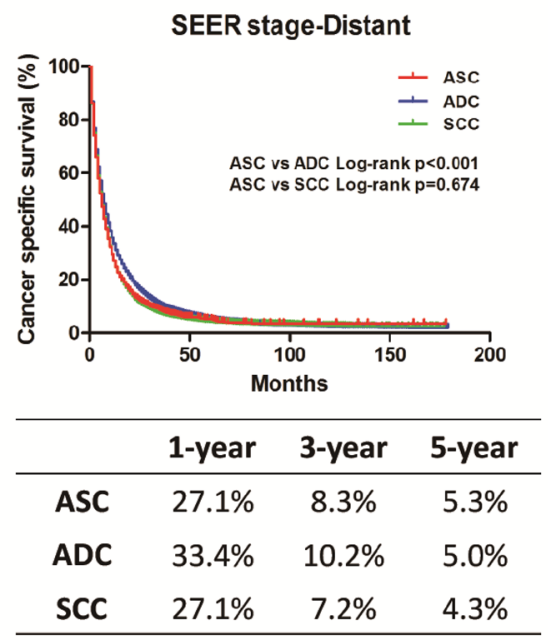

Figure 2: Kaplan-Meier plot and log-rank test for the cancer-specific survival (CSS) according to SEER stage among these three histological types. (A) Survival curves in three groups with localized disease. ASC vs. ADC, $p=0.012$; ASC vs. SCC, $p<0.001$. (B) Survival curves in three groups with regional disease. ASC vs. ADC, $p=0.004$; ASC vs. SCC, $p<0.001$. (C) Survival curves in three groups with distant disease. ASC vs. ADC, $p<0.001$; ASC vs. SCC, $p=0.674$. 


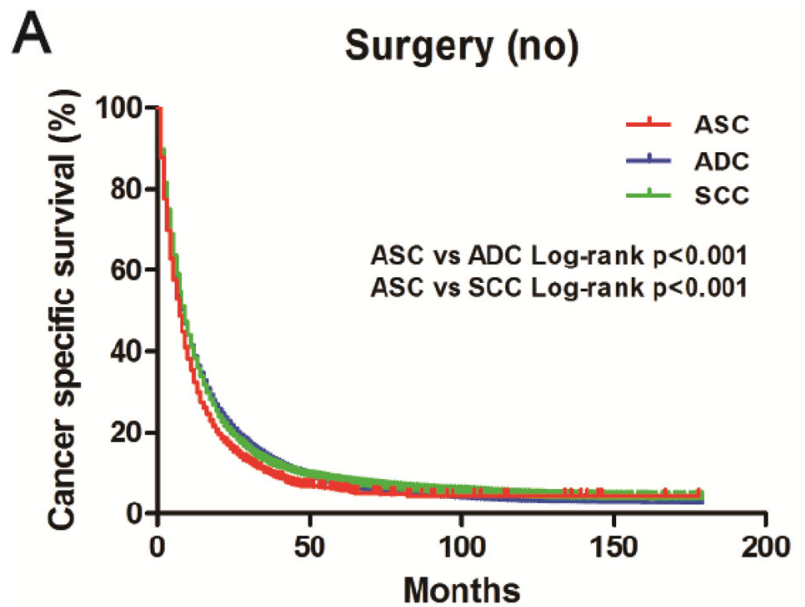

\begin{tabular}{cccc}
\hline & 1-year & 3-year & 5-year \\
\hline ASC & $32.4 \%$ & $10.1 \%$ & $5.8 \%$ \\
ADC & $38.9 \%$ & $13.5 \%$ & $7.1 \%$ \\
SCC & $38.4 \%$ & $12.8 \%$ & $7.7 \%$ \\
\hline
\end{tabular}

B Surgery (yes)

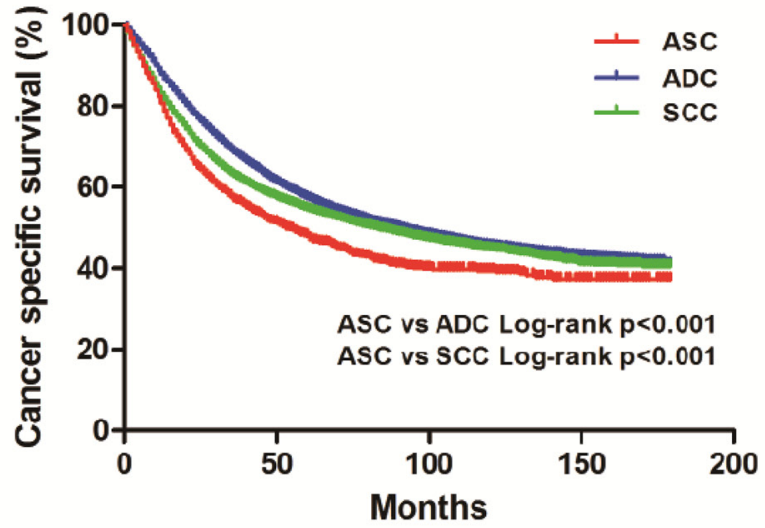

\begin{tabular}{cccc}
\hline & 1-year & 3-year & 5-year \\
\hline ASC & $80.8 \%$ & $56.8 \%$ & $47.9 \%$ \\
ADC & $88.0 \%$ & $68.6 \%$ & $57.3 \%$ \\
SCC & $83.0 \%$ & $62.8 \%$ & $54.5 \%$ \\
\hline
\end{tabular}

Figure 3: Kaplan-Meier plot and log-rank test for the cancer-specific survival (CSS) according to surgery among these three histological types. (A) Survival curves in three groups without surgery treatment. ASC vs. ADC, $p<0.001$; ASC vs. SCC, $p<$ 0.001. (B) Survival curves in three groups with surgery treatment. ASC vs. ADC, $p<0.001$; ASC vs. SCC, $p<0.001$.

A

Cancer specific survival

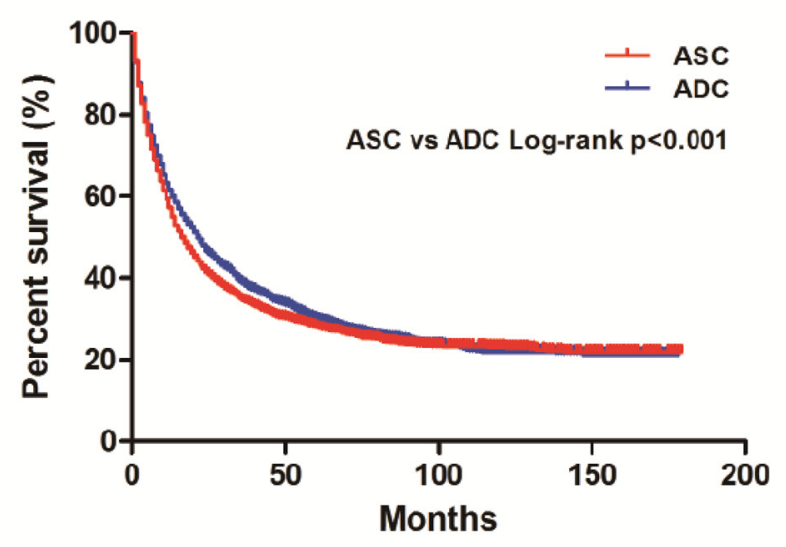

\begin{tabular}{cccc}
\hline & 1-year & 3-year & 5-year \\
\hline ASC & $57.3 \%$ & $34.6 \%$ & $28.2 \%$ \\
ADC & $61.7 \%$ & $38.8 \%$ & $30.0 \%$ \\
\hline
\end{tabular}

B

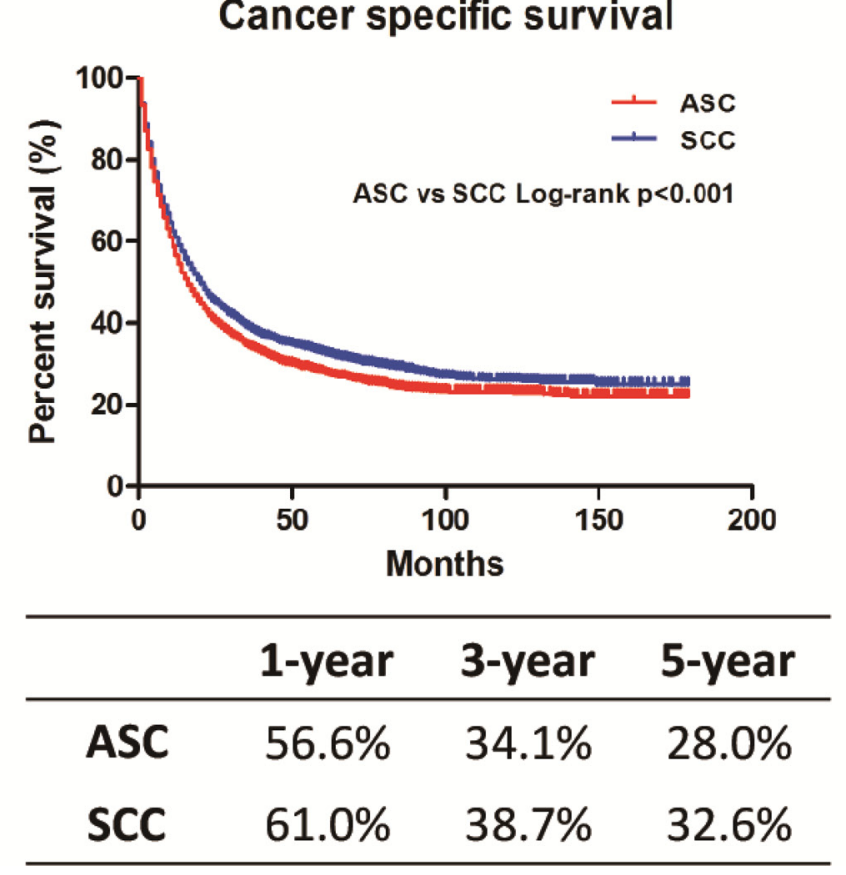

Figure 4: Kaplan-Meier plot and log-rank test for the cancer-specific survival (CSS) in 1:1 matched cohorts. (A) Survival curves in 1:1 matched ASC and ADC. $p<0.001$. (B) Survival curves in 1:1 matched ASC and SCC. $p<0.001$. 
Table 4A: The demographic and clinical characteristics of ASC and ADC patients in 1:1 matched group

\begin{tabular}{|c|c|c|c|}
\hline Characteristics & $\begin{array}{c}\text { ASC } \\
N=4245(\%)\end{array}$ & $\begin{array}{c}\text { ADC } \\
N=\mathbf{4 2 4 5}(\%)\end{array}$ & $p$ value \\
\hline Age & & & 0.941 \\
\hline$<60$ & $977(23.0)$ & $992(23.4)$ & \\
\hline $60-69$ & $1292(30.4)$ & $1304(30.7)$ & \\
\hline 70-79 & $1413(33.3)$ & $1388(32.7)$ & \\
\hline$\geq 80$ & $563(13.3)$ & $561(13.2)$ & \\
\hline Sex & & & 0.930 \\
\hline Female & 1896 (44.7) & $1900(44.8)$ & \\
\hline Male & $2349(55.3)$ & $2345(55.2)$ & \\
\hline Race & & & 0.603 \\
\hline White & $3481(82.0)$ & $3526(83.1)$ & \\
\hline Black & $471(11.1)$ & $447(10.5)$ & \\
\hline Others $^{b}$ & $285(6.7)$ & $266(6.3)$ & \\
\hline Unknown & $8(0.2)$ & $6(0.1)$ & \\
\hline Year of diagnosis & & & 0.967 \\
\hline 2000-2004 & $1192(28.1)$ & $1182(27.8)$ & \\
\hline 2005-2009 & $1352(31.8)$ & $1353(31.9)$ & \\
\hline 2010-2014 & $1701(40.1)$ & $1710(40.3)$ & \\
\hline Marital status & & & 0.296 \\
\hline Married & $2327(54.8)$ & $2396(56.4)$ & \\
\hline Not married $^{c}$ & $1730(40.8)$ & $1675(39.5)$ & \\
\hline Unknown & $188(4.4)$ & $174(4.1)$ & \\
\hline Grade $^{\mathrm{d}}$ & & & 0.318 \\
\hline I & $42(1.0)$ & $55(1.3)$ & \\
\hline II & $907(21.4)$ & $905(21.3)$ & \\
\hline III-IV & $2079(49.0)$ & 2020 (47.6) & \\
\hline Unknown & $1217(28.7)$ & $1265(29.8)$ & \\
\hline Tumor size (cm) & & & 0.783 \\
\hline$\leq 3$ & $1486(35.0)$ & $1509(35.5)$ & \\
\hline $3-5$ & 1164 (27.4) & $1175(27.7)$ & \\
\hline $5-7$ & $627(14.8)$ & $595(14.0)$ & \\
\hline$>7$ & $355(8.4)$ & $337(7.9)$ & \\
\hline Unknown & $613(14.4)$ & $629(14.8)$ & \\
\hline SEER stage & & & 0.821 \\
\hline Localized & $985(23.2)$ & $986(23.2)$ & \\
\hline Regional & $1572(37.0)$ & $1564(36.8)$ & \\
\hline Distant & 1634 (38.5) & $1650(38.9)$ & \\
\hline Unknown & $54(1.3)$ & $45(1.1)$ & \\
\hline Nodal status & & & 0.859 \\
\hline No & $1861(43.8)$ & $1841(43.4)$ & \\
\hline Yes & $2146(50.6)$ & $2157(50.8)$ & \\
\hline Unknown & $238(5.6)$ & $247(5.8)$ & \\
\hline Surgery & & & 0.224 \\
\hline No & $2091(49.3)$ & 2104 (49.6) & \\
\hline Yes & $2138(50.4)$ & $2114(49.8)$ & \\
\hline Unknown & $16(0.4)$ & $27(0.6)$ & \\
\hline Radiation & & & 0.947 \\
\hline No/ Unknown & $2592(61.1)$ & $2595(61.1)$ & \\
\hline Yes & $1653(38.9)$ & $1650(38.9)$ & \\
\hline Chemotherapy & & & 0.645 \\
\hline No/ Unknown & $2424(57.1)$ & $2403(56.6)$ & \\
\hline Yes & $1821(42.9)$ & $1842(43.4)$ & \\
\hline
\end{tabular}

Abbreviations: ASC, adenosquamous carcinoma; ADC, adenocarcinoma; SCC, squamous cell carcinoma; SEER, Surveillance Epidemiology and End Results database. ${ }^{a} p$ value between ASC and ADC or SCC was calculated by chi-square test, respectively.

bOthers included American Indian/Alaskan native, and Asian/Pacific islander.

'Not married included separated, single (never married), divorced, unmarried or domestic partner and widowed.

${ }^{\mathrm{d}}$ Grade I is well-differentiated; Grade II is moderately differentiated; Grade III is poorly differentiated; Grade IV is undifferentiated. 
Table 4B: The demographic and clinical characteristics of ASC and ASC patients in 1:1 matched group

\begin{tabular}{|c|c|c|c|}
\hline Characteristics & $\begin{array}{c}\text { ASC } \\
N=3926\end{array}$ & $\begin{array}{c}\text { SCC } \\
N=3926(\%)\end{array}$ & $p$ value \\
\hline Age & & & 0.986 \\
\hline$<60$ & $860(21.9)$ & 859 (21.9) & \\
\hline $60-69$ & $1220(31.1)$ & $1212(30.9)$ & \\
\hline $70-79$ & $1341(34.2)$ & $1339(34.1)$ & \\
\hline$\geq 80$ & $505(12.9)$ & $516(13.1)$ & \\
\hline Sex & & & 0.466 \\
\hline Female & $1720(43.8)$ & $1688(43.0)$ & \\
\hline Male & $2206(56.2)$ & $2238(57.0)$ & \\
\hline Race & & & 0.052 \\
\hline White & $3343(85.2)$ & $3299(84.0)$ & \\
\hline Black & $403(10.3)$ & $399(10.2)$ & \\
\hline Others ${ }^{\mathrm{b}}$ & $178(4.5)$ & $221(5.6)$ & \\
\hline Unknown & $2(0.1)$ & $7(0.2)$ & \\
\hline Year of diagnosis & & & 0.871 \\
\hline 2000-2004 & $1080(27.5)$ & $1097(27.9)$ & \\
\hline 2005-2009 & $1270(32.3)$ & $1251(31.9)$ & \\
\hline 2010-2014 & $1576(40.1)$ & $1578(40.2)$ & \\
\hline Marital status & & & 0.078 \\
\hline Married & $2172(55.3)$ & $2162(55.1)$ & \\
\hline Not married $^{c}$ & $1646(41.9)$ & $1621(41.3)$ & \\
\hline Unknown & $108(2.8)$ & $143(3.6)$ & \\
\hline Grade $^{d}$ & & & 0.881 \\
\hline I & $36(0.9)$ & $39(1.0)$ & \\
\hline II & $875(22.3)$ & 861 (21.9) & \\
\hline III-IV & 1907 (48.6) & 1938 (49.4) & \\
\hline Unknown & $1108(28.2)$ & $1088(27.7)$ & \\
\hline Tumor size (cm) & & & 0.878 \\
\hline$\leq 3$ & $1333(34.0)$ & $1341(34.2)$ & \\
\hline $3-5$ & $1111(28.3)$ & $1075(27.4)$ & \\
\hline $5-7$ & $585(14.9)$ & $583(14.8)$ & \\
\hline$>7$ & $333(8.5)$ & $338(8.6)$ & \\
\hline Unknown & $564(14.4)$ & $589(15.0)$ & \\
\hline SEER stage & & & 0.798 \\
\hline Localized & $954(24.3)$ & $938(23.9)$ & \\
\hline Regional & $1457(37.1)$ & $1443(36.8)$ & \\
\hline Distant & $1464(37.3)$ & $1500(38.2)$ & \\
\hline Unknown & $51(1.3)$ & $45(1.1)$ & \\
\hline Nodal status & & & 0.668 \\
\hline No & $1732(44.1)$ & 1715 (43.7) & \\
\hline Yes & $2003(51.0)$ & $2003(51.0)$ & \\
\hline Unknown & $191(4.9)$ & $208(5.3)$ & \\
\hline Surgery & & & 0.650 \\
\hline No & $2044(52.1)$ & $2023(51.5)$ & \\
\hline Yes & $1873(47.7)$ & $1897(48.3)$ & \\
\hline Unknown & $9(0.2)$ & $6(0.2)$ & \\
\hline Radiation & & & 0.908 \\
\hline $\mathrm{No} /$ Unknown & $2401(61.2)$ & $2396(61.0)$ & \\
\hline Yes & $1525(38.8)$ & $1530(39.0)$ & \\
\hline Chemotherapy & & & 0.326 \\
\hline No/ Unknown & $2283(58.2)$ & $2240(57.1)$ & \\
\hline Yes & $1643(41.8)$ & $1686(42.9)$ & \\
\hline
\end{tabular}

Abbreviations: ASC, adenosquamous carcinoma; ADC, adenocarcinoma; SCC, squamous cell carcinoma; SEER, Surveillance Epidemiology and End Results database. ${ }^{\mathrm{a}} p$ value between ASC and ADC or SCC was calculated by chi-square test, respectively.

${ }^{b}$ Others included American Indian/Alaskan native, and Asian/Pacific islander.

'Not married included separated, single (never married), divorced, unmarried or domestic partner and widowed.

'Grade I is well-differentiated; Grade II is moderately differentiated; Grade III is poorly differentiated; Grade IV is undifferentiated. 
$p=0.910)$, Grade II $(\mathrm{HR}=1.13,95 \%$ CI $0.98-1.30$, $p=0.083)$, tumor size $>7 \mathrm{~cm}(\mathrm{HR}=1.08,95 \%$ CI 0.90 $1.30, p=0.385)$, and localized subgroup $(\mathrm{HR}=1.04,95 \%$ CI $0.89-1.22, p=0.612$ ).

\section{DISCUSSION}

The present study identified 4,245 ASC patients from the SEER database and this was the largest number of patients identified compared with other studies. Through comparative analysis, we found that ASC patients shared different demographic and clinical characteristics from ADC and SCC patients. ASC patients carried a better prognosis than $\mathrm{ADC}$ and $\mathrm{SCC}$ patients. Interestingly, after 1:1 matching ASC with ADC or SCC using PSM, ASC patients had a worse prognosis than ADC and SCC patients. Furthermore, multivariate and subgroup analysis supported the ASC histology type to be an independent risk factor for the prognosis compared with ADC and SCC histology types.

The demographic and clinical characteristics of ASC were varying and inconsistent in current studies. With regard to demographic characteristics, Cooke et al. [8] analyzed early data from the SEER database (1998-2002) and showed that age and the male/female ratio of ASC lay between that of ADC and SCC. Fewer ASC patients were white race compared with $\mathrm{ADC}$ and SCC patients. In a relatively large study undertaken in Japan by Maeda and colleagues, the mean age of 114 enrolled ASC patients was lower than that of ADC and SCC patients. The male/ female ratio of ASC patients was higher than that of ADC patients, but lower than that of SCC patients [5]. A French study showed that, in 141 ASC patients from two centers, age, male/female ratio and percentage of smoking were similar to those in ADC patients, but were distinctly lower than those in SCC patients [4]. A retrospective study carried out in China evaluated 72 ASC patients, and found $63.7 \%$ of them to be male with a median age of 60 years, and that $55.6 \%$ had smoking history [13]. In another study that identified $127 \mathrm{ASC}$ patients, the male/female ratio and percentage of smokers were even higher to $72 \%$ and $90 \%$, respectively [14]. Our results were closer to those of the study by Cooke and colleagues. In our study, the age, male/ female ratio and race of ASC patients were intermediate between ADC and SCC patients. The reason for such obvious differences between our study and those of others may lie in small study cohorts or misclassification of patients for other studies. Besides, we included, for the first time, the marital status for ASC patients. We found a similar proportion of married/unmarried in ASC patients with that in ADC patients, but more SCC patients were unmarried.

With regard to the clinical characteristics, Cooke et al. [8] showed that the tumor grade of ASC patients was higher than that of ADC and SCC patients. The tumor size and prevalence of nodal metastasis for ASC patients
A

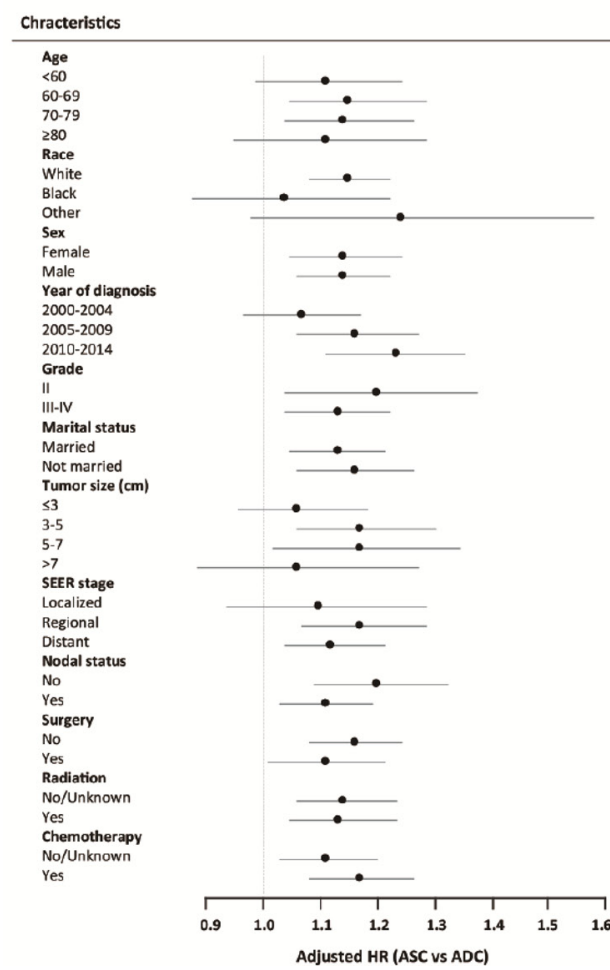

B

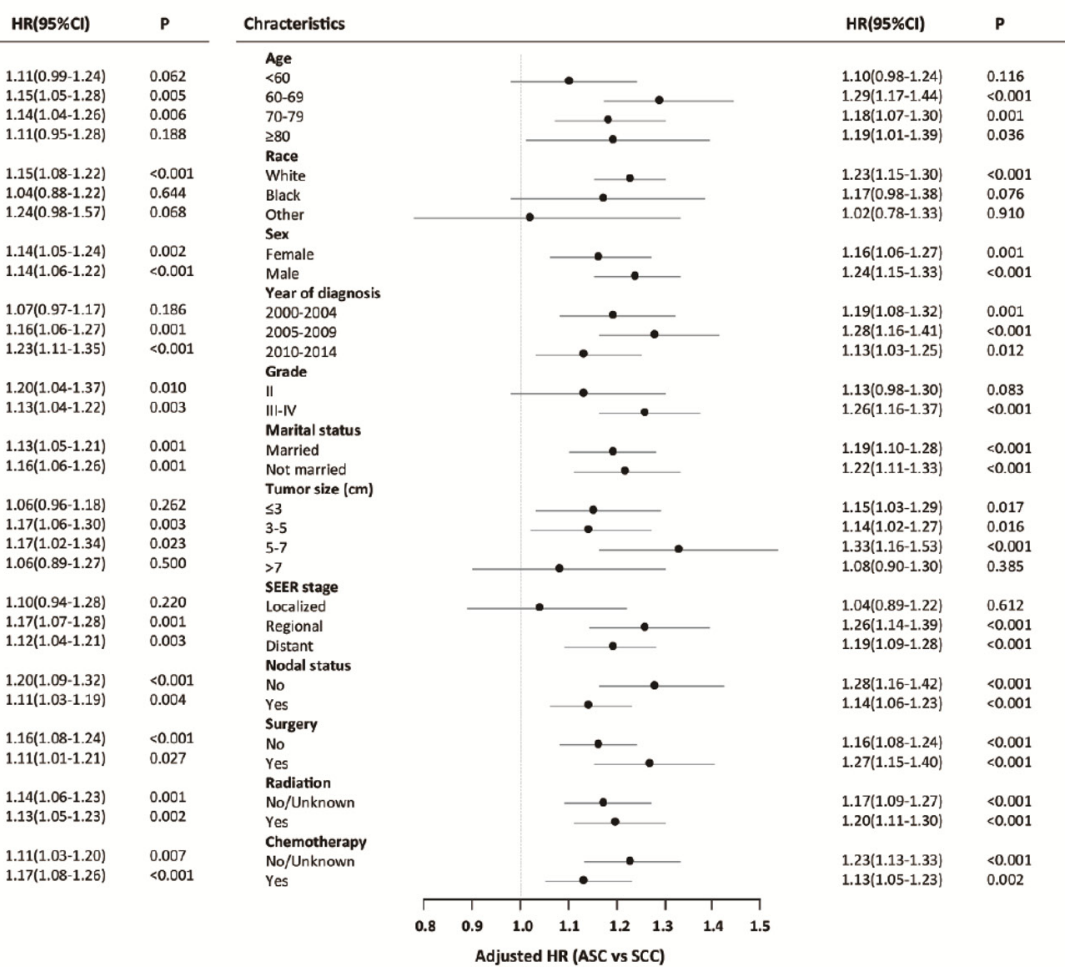

Figure 5: Forest plot of hazard ratios (HRs) for ASC versus ADC or SCC in the subgroup analysis. The circle and line segment represent the HR and 95\% confident interval of each subgroup. An HR $>1.00$ indicates higher risk for CSS in patients with ASC compared with ADC or SCC and vice versa. 
were intermediate between ADC and SCC. Filosso et al. [6] concluded, from a small number of ASC patients, that the tumor stage was higher in ASC patients compared with ADC and SCC cases. Similarly, Ruffini et al. [15] and Cakir et al. [16] concluded that ASC presented at a more advanced stage than ADC and SCC. In the study by Maeda and colleagues, ASC patients had a lower tumor grade, a larger tumor size, and higher proportions of nodal metastasis and stage IIIA compared with ADC patients, but there was no significant difference between ASC and SCC patients. Besides, the proportion of patients who underwent chemotherapy in these three groups was not significantly different [5]. Mordant et al. [4] showed that ASC had the largest tumor size among these three groups. In the present study, ASC patients had a higher tumor grade than ADC and SCC patients, which was consistent with those of Cooke et al. but different to those of Maeda and coworkers. In terms of tumor size, ASC lay between $\mathrm{ADC}$ and $\mathrm{SCC}$ in our study. Interestingly, more ASC patients were diagnosed at an early stage, compared with ADC and SCC cases. The proportion of nodal metastasis in ASC patients was slightly lower than that in $\mathrm{ADC}$ and SCC patients. With regard to treatment (surgery, radiation and chemotherapy), we evaluated ASC in a large population for the first time. We found that more ASC patients underwent surgery, compared with ADC and SCC patients. The proportion of patients who underwent radiation treatment for ASC was lower than that for SSC, and the proportion receiving chemotherapy was lower for ASC than that for ADC.

Most studies have suggested that ASC patients had worse survival than ADC and ASC patients [5-8, 17]. However, Uramoto et al. [9] showed that the prognosis of ASC patients was similar to that of ADC and ASC patients, which was in accordance with the work of Hsia et al. [10]. Interestingly, we found that the prognosis of ASC was better than that of ADC and SCC, but stratified analysis did not support this result and showed that survival for ASC patients was worse than that for ADC and SCC in the surgery and non-surgery subgroup. Multivariate analysis showed that ASC was an independent risk factor for the prognosis. Considering the effect of confounding factors on survival outcomes, we originally used a PSM to $1: 1$ match ASC with ADC or SCC. As expected, ASC patients had worse survival compared with ADC or SCC cases. The same result was found in multivariate analysis for matched groups. In subgroup analysis, ASC was identified to be an independent factor for a poor prognosis in most subgroups. Moreover, CSS at 1, 3 and 5 years for unmatched or matched ASC patients were obviously lower than that reported previously [8]. This contradiction may have been caused by early-stage disease and surgery treatment in ASC patients enrolled in most previous studies. We further confirmed that stage and surgery treatment were significantly associated with the survival of ASC patients, and were identified independent risk factors for the prognosis in univariate and multivariate analysis.

The current treatment strategy for ASC is limited and has developed mainly from studies on ADC and SCC. The prevalence of the epidermal growth factor receptor (EGFR) mutation in ASC has been reported to affect $15.4 \%$ to $44.0 \%$ of patients, which was calculated from a limited number of cases [18-21]. However, EGFR-tyrosine kinase inhibitors have been demonstrated to be effective treatment for ASC patients with the EGFR mutation [22]. In the present study, data on the EGFR mutation were not provided by the SEER database, but other significant findings were identified to assist with clinical decision for ASC treatment. In the multivariate analysis, surgery and chemotherapy, but not radiation, were protective factors for the prognosis in ASC patients. Surgery treatment for ASC patients could obviously improve survival, but the prognosis was still worse than that for ADC and SCC patients in surgery subgroup. Additionally, subgroup analysis showed that ASC contributed to worse survival compared with ADC or SCC. Thus, it reminded clinical physicians to take a more positive treatment strategy for ASC patients.

Besides, the present study had several limitations. Firstly, data on smoking history, self-reported information from patients, laboratory tests and imaging were not provided by the SEER database. Secondly, AJCC stage and metastasis site were excluded due to its inadequate information. Additionally, the detailed chemotherapy regimens and information for targeted drug were not provided by SEER database. The lack of these information may affect the survival analysis. Lastly, gene mutation data of patients (including those of the EGFR, KRAS, and BRAF mutation) were not provided by the SEER database which reduced the importance of our investigation in terms of clinical application [23].

In summary, we found $\mathrm{ASC}$ to be a unique histological type in lung cancer. It was intermediate between $\mathrm{ADC}$ and $\mathrm{SCC}$ with regard to age, sex, race, year of diagnosis, tumor size and SEER stage. Compared with $\mathrm{ADC}$ and $\mathrm{SCC}$, ASC patients presented with a higher tumor grade and lower prevalence of nodal metastasis. More ASC patients underwent surgery and a lower proportion underwent radiation treatment and chemotherapy. The prognosis of ASC patients was worse than that of ADC and SCC patients after adjustment for baseline characteristics. Multivariate and subgroup analysis supported the notion that the ASC histology type was an independent factor for a poor prognosis. These results from a large cohort of ASC patients provided a deep insight to ASC and contributed to diagnosis and treatments of ASC for clinical physicians.

\section{Author contributions}

YLS and JH provided the concept and designed the study; JW extracted the data from SEER database; JW, 
BL and LY calculated and analyzed the data; JW and BL prepared the figures and tables and wrote the manuscript. YLS, JH and LY revised the manuscript.

\section{CONFLICTS OF INTEREST}

The authors have declared that they have no competing interests.

\section{FUNDING}

This research was supported by the State Key Basic Research Program (973) project (2015CB553404), Doctoral Fund of Ministry of Education of China (20130071110044), Shanghai Science and Technology Committee (15DZ1930600/15DZ1930602), Shanghai Municipal Commission of Health and Family Planning (201540370) , National Natural Science Foundation of China (81490533), and National Natural Science Foundation of China (81500026).

\section{REFERENCES}

1. Shimoji M, Nakajima T, Yamatani C, Yamamoto M, Saishou S, Isaka M, Maniwa T, Ode Y, Nakagawa K, Okumura T, Watanabe R, Ito I, Kameya T, et al. A clinicopathological and immunohistological re-evaluation of adenosquamous carcinoma of the lung. Pathol Int. 2011; 61:717-722.

2. Travis WD, Brambilla E, Müller-Hermelink HK, Harris CC. World health organization classification of tumours. Pathology and genetics of tumours of the lung, pleura, thymus and heart. (Lyon: IARC Press) 2004.

3. Rao N. Adenosquamous carcinoma. Semin Diagn Pathol. 2014; 31:271-277.

4. Mordant P, Grand B, Cazes A, Foucault C, Dujon A, Le Pimpec BF, Riquet M. Adenosquamous carcinoma of the lung: surgical management, pathologic characteristics, and prognostic implications. Ann Thorac Surg. 2013; 95:1189-1195.

5. Maeda H, Matsumura A, Kawabata T, Suito T, Kawashima O, Watanabe T, Okabayashi K, Kubota I. Adenosquamous carcinoma of the lung: surgical results as compared with squamous cell and adenocarcinoma cases. Eur $\mathrm{J}$ Cardiothorac Surg. 2012; 41:357-361.

6. Filosso PL, Ruffini E, Asioli S, Giobbe R, Macri L, Bruna MC, Sandri A, Oliaro A. Adenosquamous lung carcinomas: a histologic subtype with poor prognosis. Lung Cancer. 2011; 74:25-29.

7. Gawrychowski J, Brulinski K, Malinowski E, Papla B. Prognosis and survival after radical resection of primary adenosquamous lung carcinoma. Eur J Cardiothorac Surg. 2005; 27:686-692.

8. Cooke DT, Nguyen DV, Yang Y, Chen SL, Yu C, Calhoun RF. Survival comparison of adenosquamous, squamous cell, and adenocarcinoma of the lung after lobectomy. Ann Thorac Surg. 2010; 90:943-948.
9. Uramoto H, Yamada S, Hanagiri T. Clinicopathological characteristics of resected adenosquamous cell carcinoma of the lung: risk of coexistent double cancer. J Cardiothorac Surg. 2010; 5:92.

10. Hsia JY, Chen CY, Hsu CP, Shai SE, Wang PY. Adenosquamous carcinoma of the lung. Surgical results compared with squamous cell and adenocarcinoma. Scand Cardiovasc J. 1999; 33:29-32.

11. Watanabe Y, Tsuta K, Kusumoto M, Yoshida A, Suzuki K, Asamura H, Tsuda H. Clinicopathologic features and computed tomographic findings of 52 surgically resected adenosquamous carcinomas of the lung. Ann Thorac Surg. 2014; 97:245-251.

12. Ren Y, Dai C, Zheng H, Zhou F, She Y, Jiang G, Fei K, Yang P, Xie D, Chen C. Prognostic effect of liver metastasis in lung cancer patients with distant metastasis. Oncotarget. 2016; 7:53245-53253. https://doi.org/10.18632/ oncotarget. 10644.

13. Wu X, Li J, Chen S, Yu L, Yang B. [Clinicopathologic Features and Prognostic Implications in 72 Cases with Lung Adenosquamous Carcinoma]. [Article in Chinese]. Zhongguo Fei Ai Za Zhi. 2016; 19:653-658.

14. Sridhar KS, Bounassi MJ, Raub WJ, Richman SP. Clinical features of adenosquamous lung carcinoma in 127 patients. Am Rev Respir Dis. 1990; 142:19-23.

15. Ruffini E, Rena O, Oliaro A, Filosso PL, Bongiovanni M, Arslanian A, Papalia E, Maggi G. Lung tumors with mixed histologic pattern. Clinico-pathologic characteristics and prognostic significance. Eur J Cardiothorac Surg. 2002; 22:701-707.

16. Cakir E, Demirag E, Aydin M, Unsal E. Clinicopathologic features and prognostic significance of lung tumours with mixed histologic patterns. Acta Chir Belg. 2009; 109:489-493.

17. Nakagawa K, Yasumitu T, Fukuhara K, Shiono H, Kikui M. Poor prognosis after lung resection for patients with adenosquamous carcinoma of the lung. Ann Thorac Surg. 2003; 75:1740-1744.

18. Kang SM, Kang HJ, Shin JH, Kim H, Shin DH, Kim SK, Kim JH, Chung KY, Kim SK, Chang J. Identical epidermal growth factor receptor mutations in adenocarcinomatous and squamous cell carcinomatous components of adenosquamous carcinoma of the lung. Cancer. 2007; 109:581-587.

19. Sasaki H, Endo K, Yukiue H, Kobayashi Y, Yano M, Fujii Y. Mutation of epidermal growth factor receptor gene in adenosquamous carcinoma of the lung. Lung Cancer. 2007; 55:129-130.

20. Toyooka S, Yatabe Y, Tokumo M, Ichimura K, Asano H, Tomii K, Aoe M, Yanai H, Date H, Mitsudomi T, Shimizu N. Mutations of epidermal growth factor receptor and K-ras genes in adenosquamous carcinoma of the lung. Int $\mathrm{J}$ Cancer. 2006; 118:1588-1590.

21. Jia XL, Chen G. EGFR and KRAS mutations in Chinese patients with adenosquamous carcinoma of the lung. Lung Cancer. 2011; 74:396-400. 
22. Song Z, Lin B, Shao L, Zhang Y. Therapeutic efficacy of gefitinib and erlotinib in patients with advanced lung adenosquamous carcinoma. J Chin Med Assoc. 2013; $76: 481-485$.
23. Park SJ, More S, Murtuza A, Woodward BD, Husain H. New Targets in Non-Small Cell Lung Cancer. Hematol Oncol Clin North Am. 2017; 31:113-129. 